\title{
Gastric trichobezoar: Food for thought
}

\author{
N Maharaj, P Naidoo, V Naidu, J Maharajh
}

Department of Diagnostic Radiology, Nelson R Mandela School of Medicine, University of Kwa-Zulu Natal, and King Edward VIII Hospital, Durban N Maharaj, MB ChB, FCRad Diag (SA)

P Naidoo, MB BCh, FCRad Diag (SA)

V Naidu, MB ChB, FCRad Diag (SA)

J Maharajh, MB ChB, FFRad (D) (SA), MMed Rad (D)

Corresponding author: N Maharaj (narishamaharaj@hotmail.com)

A bezoar is an agglomeration of food or indigestible foreign material in the gastro-intestinal tract. It is an uncommon cause of abdominal
symptomatology and can result in intestinal obstruction. This report is of an adolescent girl with a history of abdominal distension and trichophagia.

S Afr J Rad 2013;17(1):19-20. DOI:10.7196/SAJR.760

A 16-year-old girl with no past medical or surgical history presented with a 3-month history of abdominal pain and distension with weight loss. Computerised tomography (CT) scans (Figs 1 and 2) of the abdomen revealed a well-defined heterogeneous intraluminal gastric mass with interspersed air bubbles conforming to the shape of the stomach. No further intestinal lesions were noted. A diagnosis of gastric bezoar was made. A trichobezoar was confirmed on endoscopy, with subsequent successful surgical removal. A history of trichophagia was elicited post-surgery and the patient was subsequently referred for psychiatric evaluation and supportive psychotherapy to treat the impulse control disorder and prevent bezoar recurrence.

\section{Discussion}

Bezoars are agglomerations of food or indigestible material in the gastro-intestinal (GI) tract. ${ }^{[1]}$ The term 'bezoar' is thought to originate from the Arabic badzehr which implies a substance that acts as an antidote or counterpoison. ${ }^{[2]}$

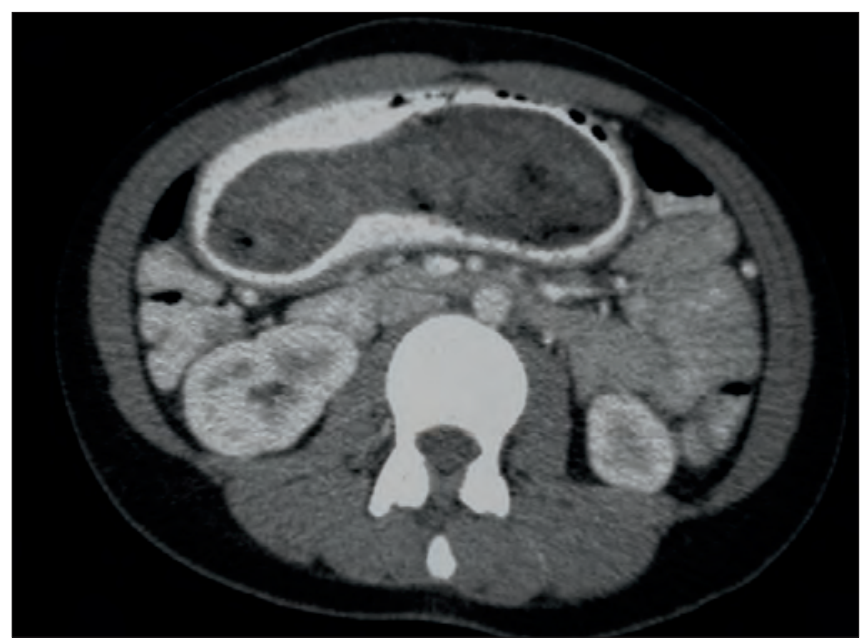

Fig. 1. Well-defined heterogeneous intraluminal gastric mass on axial postcontrast CT.
Bezoars are classified according to their primary constituent, the most common types being trichobezoars (hair) or phytobezoars (plant material). ${ }^{[3]}$ Trichobezoars occur primarily in girls who ingest their own hair, and are usually confined within the stomach. ${ }^{[3]}$

Bezoar formation is uncommon in healthy individuals and is generally associated with pica, mental retardation and psychiatric disorders. ${ }^{[1]}$ Patients are invariably teenage girls with pica or trichophagia/trichotillomania with focal alopecia. ${ }^{[2]}$ Bezoars may occur following gastric surgery such as pyloroplasty or partial gastrectomy in association with vagotomy. ${ }^{[3]}$ They may occur too in cases of delayed gastric emptying secondary to diabetes mellitus, hypothyroidism or mixed connective tissue diseases.

The clinical spectrum in GI bezoars varies, depending on the stage of bezoar formation, the length of time that it has been present, and

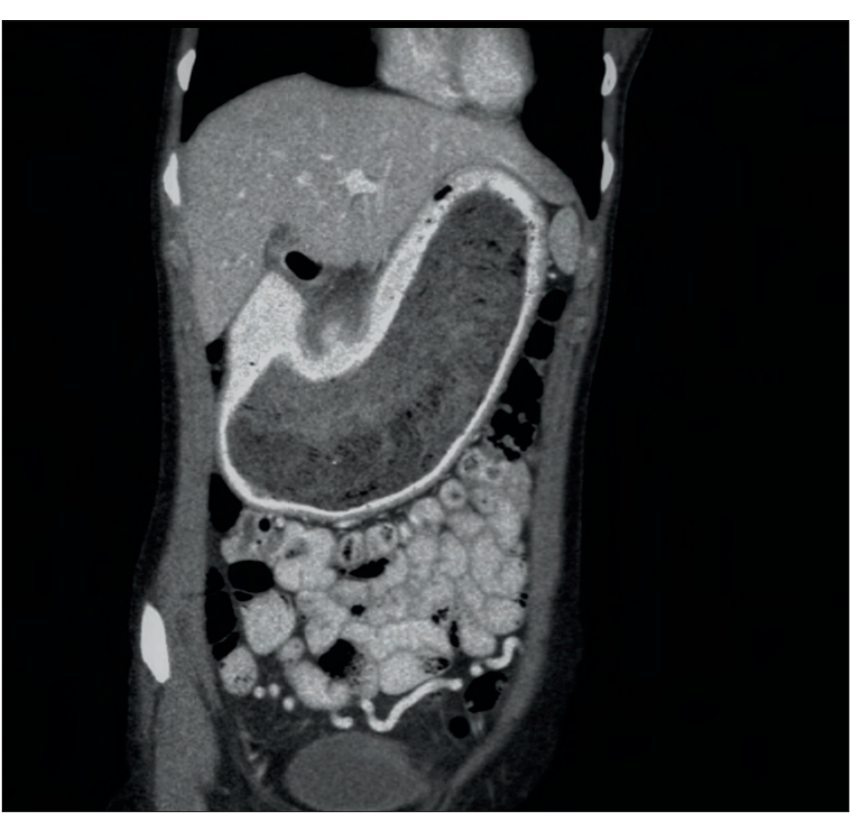

Fig. 2. Coronal post-contrast CT showing a heterogeneous intraluminal mass conforming to the configuration of the stomach with intrinsic interspersed air loculi outlined by oral contrast. 


\section{CASE REPORT}

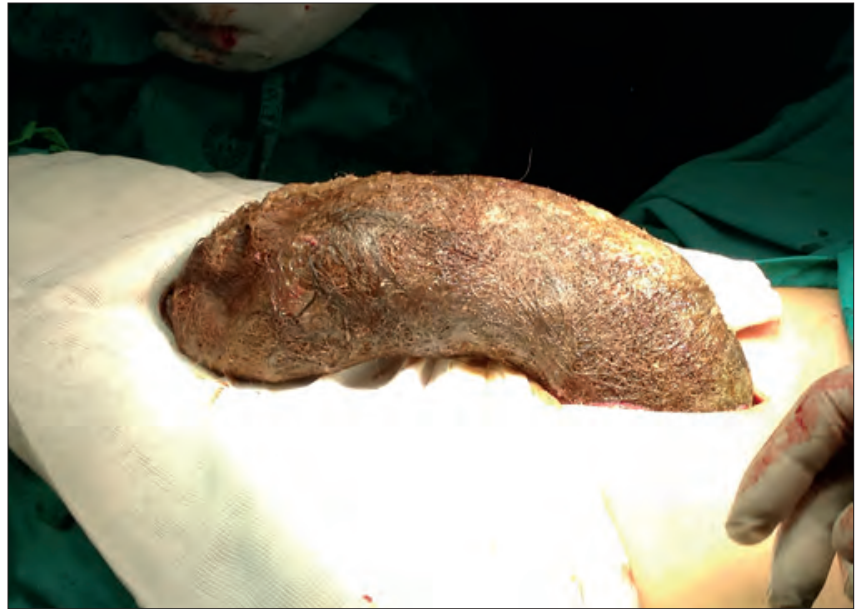

Fig. 3. Specimen retrieved at surgery.

the location within the GI tract. ${ }^{[4]}$ Common clinical features are: abdominal pain/mass, intestinal obstruction, weight loss, poor appetite or vomiting. ${ }^{[1]}$ As the bezoar enlarges, it causes compression and mechanical irritation of the intestinal mucosa, leading to complications such as chronic inflammation, ulceration, perforation, intussusception and obstruction. ${ }^{[1]}$ Intramural pneumatosis and emphysematous gastritis have been reported in association with gastric bezoars.

The Rapunzel syndrome is a rare clinical entity where a long tail of hair strands from the gastric trichobezoar extends along the small bowel to reach the caecum and beyond. There have been only 13 published cases of this entity. ${ }^{[1,2,4]}$

\section{Imaging findings}

At conventional radiography, the characteristic appearance of a gastric trichobezoar is mottled soft-tissue opacity in the shape of a distended stomach. A calcified rim may be visualised. ${ }^{[1,4]}$

Fluoroscopic studies may outline a large mottled intraluminal filling defect in the stomach and duodenum that may extend into the small bowel with barium often trapped within the interstices. ${ }^{[2]}$ There may be additional filling defects throughout the bowel owing to breakage of the bezoar. ${ }^{[4]}$ Bezoars may be mobile.

Ultrasonic appearance is that of curvilinear bright echogenic bands with posterior acoustic shadowing with no through-transmissions. Ultrasonography is, however, of limited sensitivity in revealing gastric bezoars. ${ }^{[1,3,4]}$

CT reveals a well-defined mesh-like oval intraluminal mass of low attenuation with air bubbles/oral contrast retained within the interstices.
The intraluminal mass may be outlined by orally administered contrast. ${ }^{[1,4]}$ Occasionally, it may be difficult to differentiate a bezoar from retained food; however, bezoars tend to be round/oval or fill the lumen with air bubbles distributed diffusely through the mass. ${ }^{[3]}$ Complications such as perforation, obstruction, intussusception and abscess formation may be demonstrated on CT. ${ }^{[4]}$

The MRI appearance varies depending on the content of air, fat, water or retained food debris within a gastric bezoar. ${ }^{[4]}$ Bezoars may demonstrate low signal intensity on T1, T2 and fat-suppressed T2 images owing to interspersed air loculi, or they may show high signal intensity on T1- and T2-weighted images because of food debris and water within the bezoar. Focal areas of fat within the lesion may be high signal on $\mathrm{T} 1$ and inversion recovery sequences. ${ }^{[4]}$ The bezoar does not enhance post-gadolinium administration. These techniques are expensive, time-consuming and generally less useful because bezoars can be easily confused with air on MRI owing to their signal characteristics. ${ }^{[2]}$

\section{Treatment}

The primary treatment is surgical removal. Gastrostomy/enterotomy is the modality of choice for removal of large solid bezoars; however, preliminary endoscopy is advised to confirm the diagnosis. ${ }^{[2]}$ Newer techniques with variable success rates include dormia basket extraction, direct large channel endosopic fragmentation and aspiration, bedside Coca Cola lavage, water jet, forceps and polypectomy snare.$^{[2]}$ Endoscopic retrieval of smaller lesions, extracorporeal shock wave lithotripsy, and endoscopy with utilisation of laser ignition or fragmentation with a bezotome have been suggested as alternative therapies. ${ }^{[1,4]}$ Of paramount importance to prevent recurrence is correction of the underlying impulse control disorder via behavioural training, pharmacotherapy and regular follow-up. ${ }^{[2]}$

\section{Conclusion}

Trichobezoars are an uncommon cause of abdominal symptoms in Africa; however, they are well documented in the literature. In the past, bezoars were rarely diagnosed prior to surgery, but advances in imaging suggest that ultrasonography and CT can help radiologists to diagnose bezoars prior to surgery, which improves clinical management. ${ }^{[3]}$

1. O’ Sullivan MJ, McGreal G, Walsh JG, Redmond HP. Trichobezoar. J R Soc Med 2001;94:68-70.

2. Sharma AK, Vyas CS, Porwal S. Bezoars - A variety of presentations in tricobezoars. Int J Biol Med Res 2012;3(3):2287-2292.

3. Ripolles T, Garcia-Aguayo J, Martinez MJ, Gil P. Gastrointestinal bezoars sonographic and CT characteristics. AJR 2001;177(1):65-69.

4. Koplewitz BZ, Daneman A, Fields S, Udassin R, Ein SH. Case 29: Gastric trichobezoar and subphrenic abscess. Radiology 2000;217:739-742. 